\title{
NOTE ON THE INTEGRABILITY OF SUPERHARMONIC FUNCTIONS
}

\author{
NORIAKI SUZUKI
}

(Communicated by J. Marshall Ash)

\begin{abstract}
Let $D$ be a domain in $\mathbf{R}^{n}$ and let $S^{+}(D)$ be the set of all nonnegative superharmonic functions on $D$. It is shown that if $S^{+}(D) \subset L^{p}(D)$ with some $p>0$, then for each $x_{0} \in D$ there is a constant $C=C\left(D, p, x_{0}\right)>0$ such that the inequality

$$
\int_{D} u(x)^{p} d x \leq C u\left(x_{0}\right)^{p}
$$

holds for all $u \in S^{+}(D)$.
\end{abstract}

Let $D$ be a domain in $\mathbf{R}^{n}, n \geq 2$. Denote by $S^{+}(D)$ the set of all nonnegative superharmonic functions on $D$ and by $L^{p}(D), 0<p<\infty$, the space of $p$ th integrable functions on $D$ with respect to the $n$-dimensional Lebesgue measure $d x$. Our concern is the following problem: For what domains $D$ does there exist a number $p>0$ such that

$$
S^{+}(D) \subset L^{p}(D)
$$

holds? This global integrability of superharmonic functions was first studied by Armitage [1, 2] for a bounded domain with bounded curvature and then by Maeda and Suzuki [5] for Lipschitz domains. Recently, Masumoto [6] and Smith and Stegenga [7] discussed this property for a large class of plane domains and for Hölder domains, respectively. We remark that in order to show the inclusion (1), they all proved the following inequality: For each domain $D$ under consideration, one can choose a number $p>0$ satisfying

$$
\int_{D} u(x)^{p} d x \leq C u\left(x_{0}\right)^{p} \quad \forall u \in S^{+}(D)
$$

with some constant $C=C\left(D, p, x_{0}\right)>0\left(x_{0} \in D\right.$ : arbitrarily fixed $)$. It is clear that (2) implies (1). In this article we shall prove the converse implication. Namely, we have the following

Received by the editors June 3, 1991 and, in revised form, September 18, 1991.

1991 Mathematics Subject Classification. Primary 31B05.

This research was partially supported by Grant-in-Aid for Encouragement of Young Scientist (No. 03740079) of Ministry of Education of Japan. 
Theorem. Let $D$ be a domain in $\mathbf{R}^{n}$ and $p>0$. If $S^{+}(D) \subset L^{p}(D)$, then for each $x_{0} \in D$ there is a constant $C=C\left(D, p, x_{0}\right)$ such that the inequality

$$
\int_{D} u(x)^{p} d x \leq C u\left(x_{0}\right)^{p}
$$

holds for all $u \in S^{+}(D)$.

Proof of Theorem. Let $G(\cdot, \cdot)$ denote the Green function on $D$. We consider the functional $\Phi$ on the Banach space $L^{1}(D)$ defined by

$$
\Phi(f)=\left(\int_{D} v_{f}(x)^{p} d x\right)^{1 / p}
$$

where

$$
v_{f}(x):=\int_{D} \frac{G(x, y)}{G\left(x_{0}, y\right)}|f(y)| d y .
$$

Then clearly $v_{f} \in S^{+}(D)$ so that $0 \leq \Phi(f)<\infty$. Furthermore, for any $a \in \mathbf{R}, \boldsymbol{\Phi}(a f)=|a| \Phi(f), \boldsymbol{\Phi}(f+g) \leq M_{p}(\boldsymbol{\Phi}(f)+\Phi(g))$ with some constant $M_{p}>0$, and $\liminf _{\|f-g\|_{L^{\prime}(D)} \rightarrow 0} \Phi(f) \geq \Phi(g)$. Thus the Gelfand theorem (cf. [3]) assures a constant $C>0$ such that $\Phi(f) \leq C^{1 / p}\|f\|_{L^{1}(D)}$. Since $\|f\|_{L^{1}(D)}=v_{f}\left(x_{0}\right)$, this constant $C$ brings us to inequality (3) for each $u=v_{f}$, where $f \in L^{1}(D)$ and $f \geq 0$.

Now let $v$ be a potential on the domain $D$ with compact carrier, i.e., $v=\int_{D} G(\cdot, y) d \nu(y)$ and $\nu$ is a nonnegative Borel measure on $D$ with compact support. To show (3) for $v$ we may assume that $v\left(x_{0}\right)=\int_{D} G\left(x_{0}, y\right) d \nu(y)<$ $\infty$. Then there is a sequence $\left\{f_{n}\right\}_{n=1}^{\infty}$ in $L^{1}(D)$ such that $f_{n} d y$ converges to $G\left(x_{0}, \cdot\right) d \nu$ vaguely as $n \rightarrow \infty$ and $\lim _{n \rightarrow \infty}\left\|f_{n}\right\|_{L^{1}(D)}=v\left(x_{0}\right)$. Since $\liminf _{n \rightarrow \infty} v_{f_{n}} \geq v, \int_{D} v(x)^{p} d x \leq \liminf _{n \rightarrow \infty} \int_{D} v_{f_{n}}(x)^{p} d x$ by Fatou's lemma. Hence inequality (3) for $v$ with the constant $C$ is also valid. Since every $u \in S^{+}(D)$ can be approximated by an increasing sequence of the said potentials $v$ (cf. [4, Chapter 7, §3]), we obtain (3) for all $u \in S^{+}(D)$.

3

Denote by $P(D)$ (resp. $H^{+}(D)$ ) the set of all potentials (resp. nonnegative harmonic functions) on $D$. The proof of theorem involves the following fact.

Corollary 1. If $P(D) \subset L^{p}(D)$ with some $p>0$, then $S^{+}(D) \subset L^{p}(D)$.

Modifying the preceding argument, we have the following harmonic version of our theorem.

Corollary 2. Let $p>0$. If $L^{p}(D)$ contains $H^{+}(D)$, then for each $x_{0} \in D$ there is a constant $C=C\left(D, p, x_{0}\right)>0$ such that the inequality

$$
\int_{D} h(x)^{p} d x \leq C h\left(x_{0}\right)^{p}
$$

holds for all $h \in H^{+}(D)$.

Proof. We consider the Martin boundary $X$ of $D$ (cf. [4, p. 240]). Let $K(\cdot, \cdot)$ be the Martin kernel on $D \times X$ normalized at $x_{0}$, i.e., $K\left(x_{0}, \cdot\right) \equiv 1$. Taking a 
nonnegative Borel measure $m$ on $X$ with $\operatorname{supp}(m)=X$, we define the following functional $\Psi$ on $L^{1}(X, m)$ (= the $L^{1}$ space on $X$ with respect to $m$ ):

$$
\Psi(f)=\left(\int_{D}\left(\int_{X} K(x, y)|f(y)| d m(y)\right)^{p} d x\right)^{1 / p} .
$$

Again, the Gelfand theorem gives a constant $C>0$ such that

$$
\Psi(f) \leq C\|f\|_{L^{1}(X, m)}=\int_{X} K\left(x_{0}, y\right)|f(y)| d m(y) .
$$

Now let $h \in H^{+}(D)$. Then by the Martin representation theorem [4, p. 249], there is a nonnegative Borel measure $\mu$ on $X$ such that $h=\int_{X} K(\cdot, y) d \mu(y)$. Approximating $\mu$ vaguely by a sequence $\left\{f_{n} d m\right\}_{n=1}^{\infty}$, where $f_{n} \in L^{1}(X, m)$ and $f_{n} \geq 0$, we have $h(x)=\lim _{n \rightarrow \infty} \int_{X} K(x, y) f_{n}(y) d m(y)$ and $h\left(x_{0}\right)=$ $\lim _{n \rightarrow \infty} \int_{X} f_{n}(y) d m(y)$. Thus from (5), inequality (4) for $h$ follows.

\section{REFERENCES}

1. D. H. Armitage, On the global integrability of superharmonic functions in balls, J. London Math. Soc. (2) 4 (1971), 365-373.

2. __ Further results on the global integrability of superharmonic functions, J. London Math. Soc. (2) 6 (1972), 109-121.

3. I. M. Gelfand, Sur un lemma de la théorie des espaces linéaires, Izv. Nauchno-Issled. Inst. Mat. Khar'kov Univ. Ser. 413 (1936), 35-40.

4. L. L. Helms, Introduction to potential theory, Wiley, New York, 1969.

5. F.-Y. Maeda and N. Suzuki, The integrability of superharmonic functions on Lipschitz domains, Bull. London Math. Soc. 21 (1989), 270-278.

6. M. Masumoto, Integrability of superharmonic functions on plane domains, J. London Math. Soc. (to appear).

7. W. Smith and D. A. Stegenga, Sobolev imbedding and integrability of harmonic functions on Hölder domains, Potential Theory (Proc. of ICPT 90 at Nagoya), Walter de Gruyter, Berlin, 1992, pp. 303-313.

Department of Mathematics, College of General Education, Nagoya University, NAGOYA, 464-01 JAPAN 